\title{
Optimal control theory for optical waveguide design: application to $Y$-branch structures
}

\author{
Dhruv K. Pant, Rob D. Coalson, Marta I. Hernández, and José Campos-Martínez
}

\begin{abstract}
A recently introduced optimal control theory method for optical waveguide design is applied to $Y$-branch waveguides and Mach-Zehnder modulators. The method simultaneously optimizes many parameters in a chosen design scheme; computational effort scales mildly with the number of parameters considered. Significant improvement in guiding efficiency relative to intuitively reasonable initial parameter choices is obtained in all cases. (C) 1999 Optical Society of America

OCIS codes: $230.7370,230.1360,350.5500$.
\end{abstract}

\section{Introduction}

The design of optical waveguides is of central importance to the development of efficient devices for optoelectronics applications. To ensure optimal guiding for complex waveguides (in which the complexity may arise from the details of individual guiding elements or from the existence of a network of waveguides staged in series), it is of interest to develop efficient and flexible optimization procedures based on first principles, namely, the solution of Maxwell's equations. ${ }^{1}$

The propagation of light through many waveguides is well approximated by the scalar optics approximation to Maxwell's equations, i.e., the Helmholtz equation. ${ }^{2}$ In fact, for waveguides that do not bend too severely and for which the fractional index variation inside versus outside the guiding region is small, the Helmholtz equation can be replaced by a simpler scalar wave equation, the paraxial equation. ${ }^{3}$ The paraxial equation is isomorphic to the timedependent Schrödinger equation of quantum mechanics (the role of time is played by the propagation axis coordinate; see Section 2).

In the quantum dynamics community, techniques of optimal control theory (OCT) have been utilized to

D. K. Pant and R. D. Coalson are with the Department of Chemistry, University of Pittsburgh, Pittsburgh, Pennsylvania 15260. The e-mail address for R. D. Coalson is coalson@vms.cis.pitt.edu. M. I. Hernández and J. Campos-Martínez are with the Instituto de Matemáticas y Física Fundamental, Serrano 123, Madrid E-28006 Spain.

Received 8 December 1998; revised manuscript received 18 March 1999.

0003-6935/99/183917-07\$15.00/0

(C) 1999 Optical Society of America provide an automated computational procedure for optimizing light pulses that stimulate molecular transformations, as governed by the time-dependent Schrödinger equation. ${ }^{4}$ Recently we adapted this machinery to the problem of optical waveguide design, ${ }^{5}$ exploiting the isomorphism between quantum mechanics and scalar optics as well as the framework of OCT developed in the quantum dynamics literature.

In Ref. 5 some simple numerical examples were presented to illustrate the concepts developed in that research. Specifically, we utilized the procedure to optimize the shape of a simple bend waveguide. The goal of the present paper is to demonstrate the utility of our OCT method for complex waveguide structures. The examples provided in this paper demonstrate convincingly that the procedure can be used to optimize arbitrary geometric or refractive-index features, and that the numerical cost of the method scales mildly with the number of parameters being simultaneously optimized.

We focus here on a specific wide-angle, low-loss $Y$-branch structure developed recently by Langer and co-workers. ${ }^{6,7} \quad Y$ branches play an important role in waveguiding devices, and the goal of making compact, efficient $Y$-branch structures has stimulated considerable research in recent years. ${ }^{8-11}$ Langer's compact low-loss $Y$-branch waveguide utilizes a $1 \times 2$ multimode interference (MMI) device ${ }^{12}$ and a lowered refractive index in the $Y$-junction region. ${ }^{8,10}$ (The lowering of this index is achieved by changing the height of the ridge above the waveguide, which can be incorporated easily into the fabrication process.) It was found by empirical adjustment of fabrication parameters that this device was characterized by re- 
duced radiation losses over a range of $Y$-branch angles. ${ }^{6}$

We show in the present research that, by applying our OCT scheme, radiation losses in Langer's $Y$-branch waveguide can be significantly reduced beyond the range obtained with sensibly but empirically chosen geometric and refractive-index parameters. Further calculations on MachZehnder devices based on this $Y$-branch structure lead to the same conclusion. The ability of the OCT algorithm to simultaneously optimize multiple parameters whose performance in a complex waveguide device is coupled is a strength of the method that we seek to clearly demonstrate.

The outline of this paper is as follows. In Section 2 a brief overview of the theoretical background is provided. In Section 3 the $Y$-branch waveguide under consideration is described and the parameters to be optimized are specified. In Section 4 numerical results utilizing the optimal control strategy are presented. The method is further applied to the full Mach-Zehnder modulator in Section 5. Finally, a summary and brief suggestions for future research are given in Section 6.

\section{Theoretical Background}

The paraxial equation reads ${ }^{3}$

$$
\begin{aligned}
i \frac{\partial \psi(x, y, z)}{\partial z}= & \left\{\frac{-1}{2 \beta_{0}} \nabla_{T}^{2}+\frac{k}{2 n_{0}}\left[n_{0}{ }^{2}\right.\right. \\
& \left.\left.-n(x, y, z)^{2}\right]\right\} \psi(x, y, z) .
\end{aligned}
$$

Here $\beta_{0}=k n_{0}$ with $n_{0}$ a reference refractive index (usually the index of the substrate) and $k=2 \pi / \lambda, \lambda$ being the free-space index of the light. Furthermore, $\nabla_{T}^{2} \equiv \partial^{2} / \partial x^{2}+\partial^{2} / \partial y^{2}$ is the transverse Laplacian, and $\psi$ is the slowly varying amplitude factor of the electric field. $n(x, y, z)$ describes the refractiveindex variation over the waveguide. The paraxial equation has the same form as the time-dependent Schrödinger equation ${ }^{13}$ with two spatial coordinates $(x, y)$ and the timelike coordinate $(z)$.

The two-dimensional transverse refractive-index profile can, in many cases, be reduced to an effective one-dimensional profile by the effective index method. ${ }^{14,15}$ Thus, if $n_{\text {eff }}(x, z)$ corresponds to the effective index profile in the single transverse coordinate, then the paraxial equation simplifies to

$$
i \frac{\partial \psi(x, z)}{\partial z}=\left\{\frac{-1}{2 \beta_{0}} \frac{\partial^{2}}{\partial x^{2}}+\frac{k}{2 n_{0}}\left[n_{0}{ }^{2}-n_{\mathrm{eff}}^{2}(x, z)\right]\right\} \psi(x, z) .
$$

Equation (2) has the form of the quantum-mechanical Schrödinger equation, ${ }^{13}$ namely,

$$
i \frac{\partial \psi(x, z)}{\partial z}=\hat{H}(z) \psi(x, z)
$$

with the effective Hamiltonian operator $\hat{H} \equiv \hat{T}+$ $V(x, z)$ comprised of

$$
\hat{T} \equiv \frac{-1}{2 \beta_{0}} \frac{\partial^{2}}{\partial x^{2}} ; \quad V(x, z) \equiv \frac{k}{2 n_{0}}\left[n_{0}^{2}-n_{\mathrm{eff}}^{2}(x, z)\right] .
$$

Equation 3 describes the evolution of the wave function of a particle moving along one spatial coordinate $x$ as a function of the time (equivalent to $z$ in the paraxial equation), with a kinetic energy $\hat{T}$ and a potential energy V; the latter is dependent on the position $x$ and the time (distance along the propagation axis $z$ in the paraxial equation). ${ }^{16}$ For an initial electric field amplitude $\psi(x, 0)$ at the input to the waveguide, solution of the paraxial equation provides the amplitude at all distances along the propagation axis $\psi(x, z)$. In practice the appropriate initial electric field $\psi(x, 0)$ is a guided mode, usually the fundamental mode, of the input waveguide. Such guided modes correspond to eigenfunctions of $\hat{H}(0)$, which can be computed either by expanding $\psi$ in a complete basis of orthogonal functions and solving the equivalent eigenvalue-eigenvector problem ${ }^{13}$ or by a boundary-matching method. ${ }^{2}$ Given $\psi(x, 0)$, a beam propagation method (BPM) such as the split operator technique of Feit and Fleck ${ }^{17,18}$ can be used to determine $\psi(x, z)$ along the entire waveguide.

In OCT, ${ }^{4}$ we minimize a function $J$ defined as $J[\mathbf{p}]$ $=-\langle\psi(L)|\hat{O}| \psi(L)\rangle$, where $\mathbf{p}=\left(p_{1}, p_{2}, \ldots\right)$ is the set of parameters being optimized, $L$ is the length of the waveguide, and $O$ is the relevant projection operator. ${ }^{19}$ Following Ref. 5, we maximize the overlap of the propagated field at the output $\psi(x, L)$ with a prescribed target field $\psi_{\text {target }}(x)$, which is chosen as the fundamental mode of the waveguide at its output. ${ }^{20}$ The appropriate projection operator is thus

$$
\hat{O} \equiv\left|\psi_{\text {target }}\right\rangle\left\langle\psi_{\text {target }}\right| .
$$

The corresponding cost function takes the form

$$
J[\mathbf{p}]=-\langle\psi(L)|\hat{O}| \psi(L)\rangle=-\left|\left\langle\psi_{\text {target }} \mid \psi(L)\right\rangle\right|^{2} .
$$

Once the cost function has been specified, one needs to evaluate the derivatives of $J$ with respect to each parameter, which are given as $^{5}$

$$
\frac{\partial J}{\partial p_{j}}=2 \operatorname{Re} \int_{0}^{L} \mathrm{~d} z\left\langle\lambda(z)\left|\frac{\partial H}{\partial p_{j}}\right| \psi(z)\right\rangle,
$$

where $\langle x \mid \lambda(z)\rangle=\lambda(x, z)$ is a Lagrange multiplier function introduced in the analysis. The algorithm for the computation is comprised of the following steps:

(i) $|\psi(z)\rangle$ is propagated forward from $|\psi(0)\rangle$ under the Hamiltonian operator $\hat{H}$, using a BPM.

(ii) $|\lambda(L)\rangle$ is evaluated as

$$
|\lambda(L)\rangle=-i \hat{O}|\psi(L)\rangle
$$




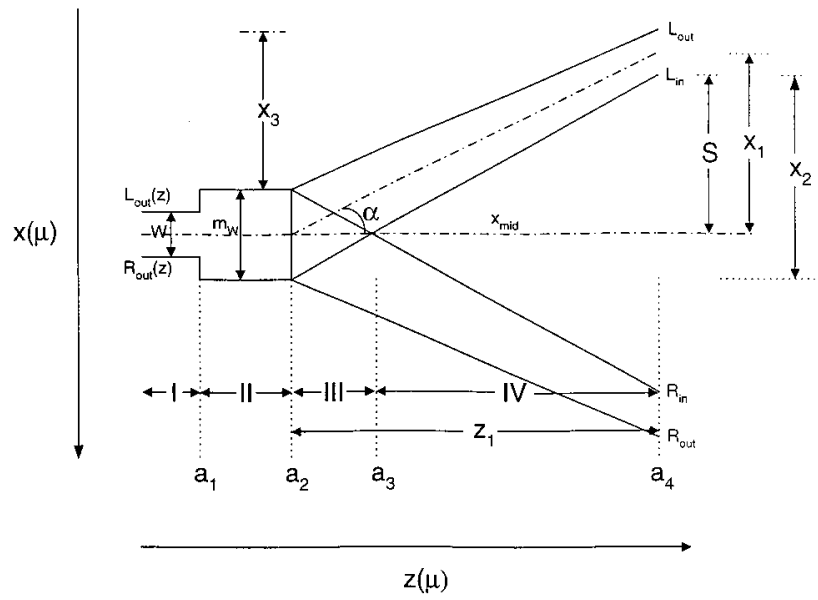

Fig. 1. Typical $Y$-branch waveguide.

(iii) $|\lambda(x, z)\rangle$ is obtained by propagating $|\lambda(L)\rangle$ backward from $z=L$ to $z=0$, using

$$
i \frac{\partial|\lambda(z)\rangle}{\partial z}=\hat{H}(z)|\lambda(z)\rangle .
$$

(iv) The gradient of $J$ is computed using Eq. (7).

(v) Finally, the method of steepest descents ${ }^{21}$ is used to locate the minimum of the function $J$.

The wave packet $\lambda(x, L)$ that is backpropagated in step (iii) depends on $O$, according to Eq. (8). In the case of interest here, where we are interested in optimization of overlap with a chosen target state, the target operator is given by Eq. (5), and therefore the specific form of the Lagrange multiplier is

$$
\lambda(x, L)=-i\left\langle\psi_{\text {target }} \mid \psi(L)\right\rangle \psi_{\text {target }}(x),
$$

i.e., the wave packet to be backpropagated is the target state function itself, multiplied by an appropriate scale factor.

\section{Details}

Figure 1 depicts the wide-angle low-loss waveguide proposed by Langer et al. ${ }^{6}$ Important ingredients are the $1 \times 2$ MMI device (region II) and the modified index in the triangular area of region III. The MMI device splits the incoming light beam into two parts, and the internal index mismatch between the triangular region and the rest of the waveguide helps to focus the two beams of light into their respective arms. The goal of our application of OCT is to simultaneously optimize the geometry (length and width) of the MMI section of the waveguide and the refractive index of the triangle junction region to minimize radiation loss down the guide arms. To demonstrate the flexibility of the algorithm, in some of our calculations we also allow the arms of the waveguide to bend (see below), rather than be perfectly straight as depicted in Fig. 1.

The angle that the symmetric $Y$ branch makes with the waveguide axis (i.e., half the Y-branch angle) is defined as $\alpha=\tan ^{-1}\left(x_{1} / z_{1}\right)$, with $x_{1} \equiv S+W / 2$ and $z_{1} \equiv\left(a_{4}-a_{2}\right)$, as indicated in Fig. 1 .

The point $a_{2}$ is kept fixed in the calculation and the MMI length is varied by changing $a_{1}$. This makes the length of region I variable. However, because the ground-state eigenmode remains unchanged over the propagation distance of region I, the length of region I is not relevant. It acts only as a buffer zone, absorbing increments and decrements of region II and thus allowing the total waveguide length to be held constant for a given $Y$-branch angle.

$m_{W}$ is the width of the MMI section. $L_{\text {out }}(z)$ and $R_{\text {out }}(z)$ define the outline of the waveguide from the left and the right, respectively, and, from region III onward, $L_{\text {in }}(z)$ and $R_{\text {in }}(z)$ define the outline of the guide on the inside. Thus in region I,

$$
\begin{aligned}
& L_{\text {out }}=x_{\text {mid }}-W / 2, \\
& R_{\text {out }}=x_{\text {mid }}+W / 2,
\end{aligned}
$$

whereas in region II,

$$
\begin{aligned}
& L_{\text {out }}=x_{\text {mid }}-m_{W} / 2=L_{\mathrm{MMI}}, \\
& R_{\text {out }}=x_{\text {mid }}+m_{W} / 2=R_{\mathrm{MMI}} .
\end{aligned}
$$

From region III on, the boundaries of the guide are expressed in general as

$$
\begin{aligned}
L_{\text {out }} & =L_{\mathrm{MMI}}-\frac{x_{3}}{z_{1}}\left(z-a_{2}\right)+\sum_{j} b_{j} \sin \left[j \pi\left(z-a_{2}\right) / z_{1}\right], \\
R_{\text {out }} & =R_{\mathrm{MMI}}+\frac{x_{3}}{z_{1}}\left(z-a_{2}\right)-\sum_{j} b_{j} \sin \left[j \pi\left(z-a_{2}\right) / z_{1}\right], \\
L_{\text {in }} & =R_{\mathrm{MMI}}-\frac{x_{2}}{z_{1}}\left(z-a_{2}\right)+\sum_{j} b_{j} \sin \left[j \pi\left(z-a_{2}\right) / z_{1}\right], \\
R_{\text {in }} & =L_{\mathrm{MMI}}+\frac{x_{2}}{z_{1}}\left(z-a_{2}\right)-\sum_{j} b_{j} \sin \left[j \pi\left(z-a_{2}\right) / z_{1}\right],
\end{aligned}
$$

with $x_{2} \equiv S+m_{W} / 2$ and $x_{3} \equiv S+W-m_{W} / 2$. In this parameterization, $b_{j}$ 's are Fourier coefficients used to describe a bend in the arms of the waveguide (straight arms are obtained when $b_{j}=0$ ).

As noted in Eq. (4), the effective potential function is written as

$$
V(x, z)=\frac{k}{2 n_{0}}\left[n_{0}{ }^{2}-n(x, z)^{2}\right],
$$

where we dropped the subscript eff from $n_{\text {eff }}(x, z)$ for notational convenience. $n^{2}$ can be expressed as a function of the set of parameters $\mathbf{p}$, which include the wedge index $\left(n_{W}\right)$, the MMI length $\left(m_{L}\right)$, the MMI width $\left(m_{W}\right)$, and the Fourier coefficients. Because

$$
\frac{\partial H}{\partial p_{j}} \equiv \frac{\partial V}{\partial p_{j}} \equiv-\frac{k}{2 n_{0}} \frac{\partial n^{2}}{\partial p_{j}},
$$

one needs expressions for $\partial n^{2} / \partial p_{j}$ to evaluate the integral in Eq. (7); these are obtained easily for the functional forms employed here. 
Table 1. Fixed Structural and Refractive-Index Parameters

\begin{tabular}{ll}
\hline \multicolumn{1}{c}{ Parameter } & Value \\
\hline$n($ cladd $) \equiv n_{2}$ & 3.558 \\
$n($ guide $) \equiv n_{1}$ & 3.568 \\
$n($ ref $) \equiv n_{0}$ & 3.558 \\
Waveguide width $(W)$ & $2 \mu \mathrm{m}$ \\
Waveguide separation $(S)$ & $4 \mu \mathrm{m}$ \\
\hline
\end{tabular}

\section{Results for the $Y$ Branch}

Structural and refractive-index parameters that were fixed (not subjected to variation) are listed in Table 1. As noted above, the width and length of the MMI device, the refractive index in the triangular wedge in region III, and, in some calculations, Fourier coefficients prescribing a bend in the arms of the waveguide were allowed to vary. Initial (guess) values were chosen based on the following considerations. A value of 3.558 for the wedge index was used for the reduced value of the index in the wedge area (equal to the index in the cladding), as suggested in Refs. 8 and 10. All Fourier coefficients were set to zero. A width of $4 \mu \mathrm{m}$ for the MMI was selected. For an incident waveguide width of $2 \mu \mathrm{m}$, this corresponds to a $1 \times 2$ converter. To determine a reasonable guess value for the length of the MMI section, we performed BPM calculations for the $Y$-branch angle $\alpha=2^{\circ}$, fixing all variable parameters at the guess values just noted except for the MMI length, which was allowed to vary. Results for the overlap of the output field with the desired target state as a function of the MMI length are shown in Fig. 2, from which it is clear that a length of $100 \mu \mathrm{m}$ is approximately optimal (gives best guiding).

BPM calculations were performed on the guess parameters identified in the previous paragraph for a range of angles $\alpha{ }^{22}$ Then the OCT procedure was used to optimize MMI width, length, and wedge index value simultaneously. (Fourier coefficients were added to the variational parameter list in subsequent calculations, described below.) It is worthwhile to

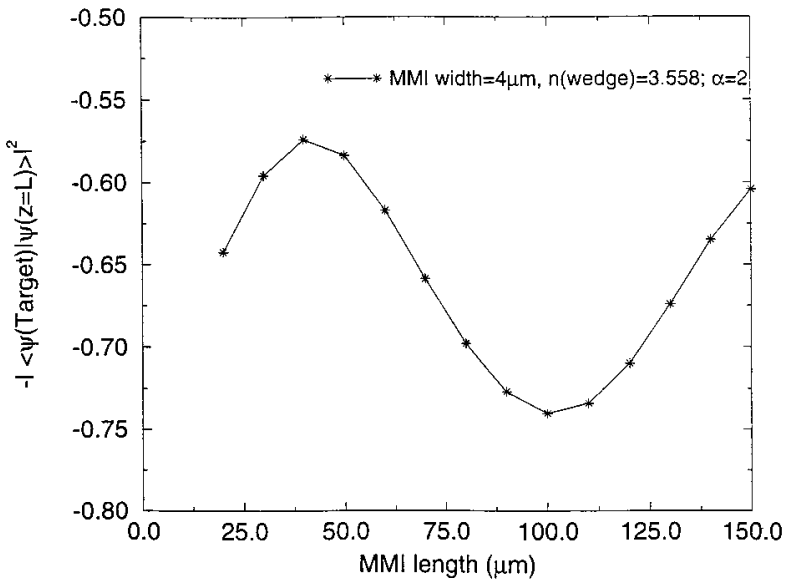

Fig. 2. Plot of $-\left|\left\langle\psi_{\text {target }} \mid \psi(z=L)\right\rangle\right|^{2}$ versus MMI length for $\alpha=2$. The fixed values of the other parameters are MMI width, $4 \mu \mathrm{m}$, and $n$ (wedge), 3.558.

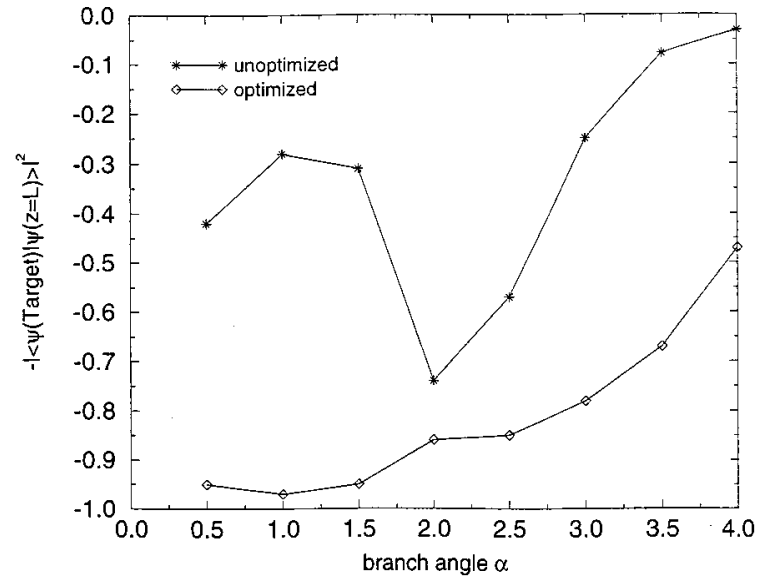

Fig. 3. $-\left|\left\langle\psi_{\text {target }} \mid \psi(z=L)\right\rangle\right|^{2}$ versus branch angle $\alpha$ for the unoptimized (upper curve) and optimized case (lower curve).

summarize the optimization procedure. Given an initial set of variational parameters $\mathbf{p}$, the gradient of the cost function $J(\mathbf{p})$ (slope in all directions of parameter space) is obtained from exactly two BPM propagations: The initial electric field amplitude is propagated forward down the beam, and the target electric field amplitude is propagated backward along the beam. The ability to provide the derivative of the cost function along an arbitrarily large number of parameter axes from only two BPM propagations is the essential strength of the OCT method developed in Ref. 5 and utilized here. Given the gradient of $J$, a small step can be taken along the negative of the direction in parameter space prescribed by the gradient vector, i.e. $p_{j} \rightarrow p_{j}-\epsilon \partial J / \partial p_{j}$, where $\epsilon$ is a small positive number. This takes us closer to the minimum of $J$; the procedure is repeated until the minimum is located. We term this the optimal control and steepest descent (OCSD) protocol.

Comparison of the quality of the beam propagation achieved with and without OCT optimization is shown in Figs. 3-7. As is easily appreciated from

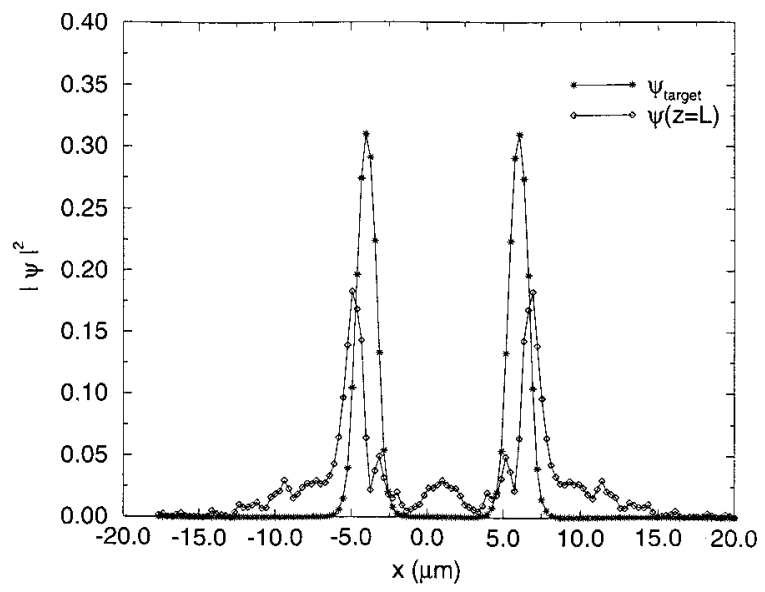

Fig. 4. Plot of the intensities of the wave function at $z=L$ (unoptimized case) and the target wave function [MMI width, $4 \mu \mathrm{m}$; MMI length, $100 \mu \mathrm{m} ; n$ (wedge), 3.558; $\alpha=1],\left|\left\langle\psi_{\text {target }} \mid \psi(z=L)\right\rangle\right|^{2}=$ 0.28 . 


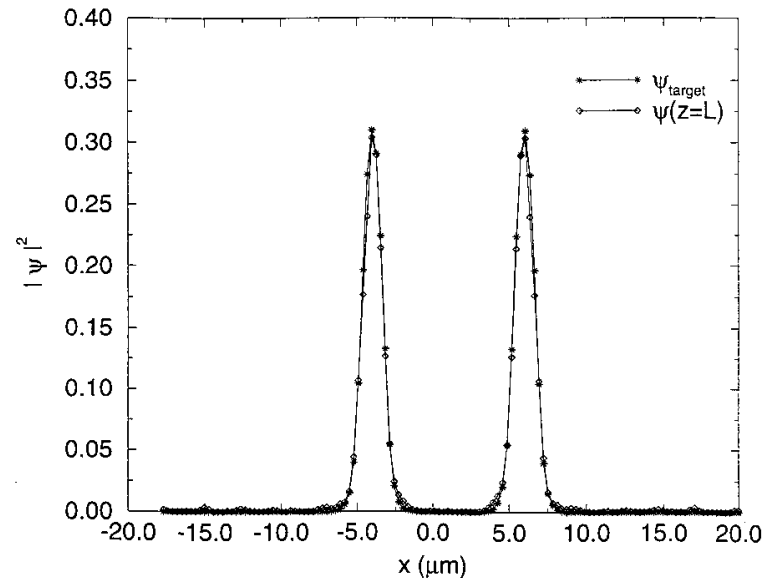

Fig. 5. Plot of the intensities of the wave function at $z=L$ (optimized case) and the target wave function [MMI width, 3.81 $\mu \mathrm{m}$; MMI length, $98.4 \mu \mathrm{m} ; n$ (wedge), 3.5662; $\alpha=1], \mid\left\langle\psi_{\text {target }}\right| \psi(z=$ $L)\rangle\left.\right|^{2}=0.97$.

these figures, OCT optimization leads to significant improvement in the performance of the waveguide at all branching angles considered. The optimized parameters corresponding to each angle are presented in Table 2.

To further demonstrate the flexibility of the OCSD protocol and the ease with which it can accommodate

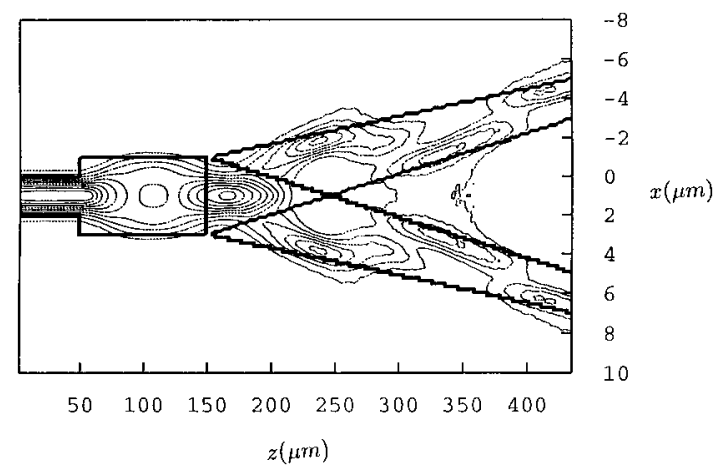

Fig. 6. Contour plots of the waveguide and the beam (unoptimized case) [MMI width, $4 \mu \mathrm{m}$; MMI length, $100 \mu \mathrm{m}$; $n$ (wedge), 3.558; $\alpha=1],\left|\left\langle\psi_{\text {target }} \mid \psi(z=L)\right\rangle\right|^{2}=0.28$.

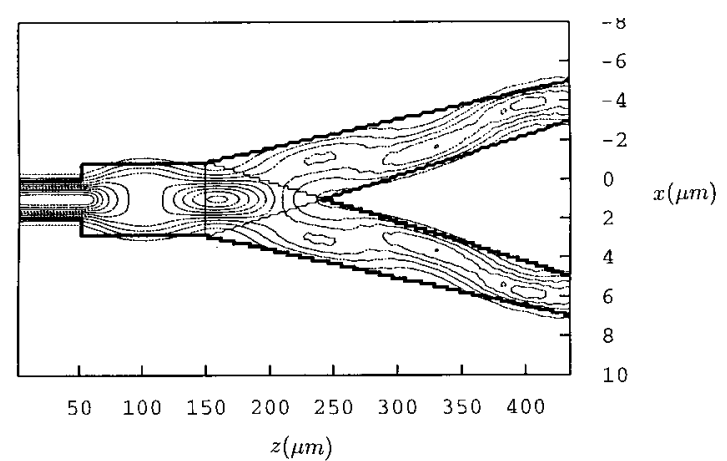

Fig. 7. Contour plots of the waveguide and the beam (optimized case) $[$ MMI width, $3.81 \mu \mathrm{m}$; MMI length, $98.4 \mu \mathrm{m} ; n$ (wedge), $3.5662 ; \alpha=1]$, $\left|\left\langle\psi_{\text {target }} \mid \psi(z=L)\right\rangle\right|^{2}=0.97$.
Table 2. Optimized Parameters

\begin{tabular}{|c|c|c|c|c|c|}
\hline$\alpha$ & $\begin{array}{l}\text { MMI } \\
\text { Width }\end{array}$ & $\begin{array}{l}\text { MMI } \\
\text { Length }\end{array}$ & $n$ (Wedge) & $\begin{array}{c}J \\
\text { (Optimized) }\end{array}$ & $\begin{array}{c}J \\
\text { (Unoptimized) }\end{array}$ \\
\hline 0.5 & 2.60 & 110.3 & 3.5657 & -0.95 & -0.42 \\
\hline 1.0 & 3.81 & 98.4 & 3.5662 & -0.97 & -0.28 \\
\hline 1.5 & 3.31 & 83.1 & 3.5695 & -0.95 & -0.30 \\
\hline 2.0 & 4.70 & 131.0 & 3.5574 & -0.86 & -0.74 \\
\hline 2.5 & 3.97 & 104.2 & 3.5495 & -0.85 & -0.57 \\
\hline 3.0 & 3.46 & 94.6 & 3.5430 & -0.78 & -0.25 \\
\hline 3.5 & 3.15 & 98.8 & 3.5341 & -0.67 & -0.08 \\
\hline 4.0 & 2.95 & 90.0 & 3.5280 & -0.47 & -0.03 \\
\hline
\end{tabular}

a large number of parameters to be simultaneously optimized, we also added to the set of variational parameters two Fourier coefficients, namely, $b_{1}$ and $b_{3}$, thus raising the total number of variational parameters in the calculation to five. ${ }^{24}$ Figure 8 shows the influence of the optimized coefficients (namely, $b_{1}=-0.15$ and $\left.b_{3}=0.16\right)$ on the shape of the waveguide. Although the improvement in the overlap integral is not significant here (for $\alpha=1$ ) compared with the optimized case with three parameters presented above, these results show that shaping of the $Y$ branch can be incorporated into the optimization procedure. As a representative case, we used the coefficients $b_{1}$ and $b_{3}$, but larger numbers of coefficients can be handled easily.

\section{Extension to Mach-Zehnder Modulators}

To further demonstrate the utility of the OCSD protocol for designing complex waveguide structures, we also applied it to Mach-Zehnder structures based on the Langer wide-angle $Y$-branch design. A typical Mach-Zehnder device with a MMI section is shown in Fig. 9. It is more complicated geometrically than a single $Y$ junction. In particular, there are more regions where radiation loss can occur, namely, at the four bends of the waveguide. As the device becomes more complex, it becomes harder to guess the optimal structure because overall performance depends on the coupled effects of many structural and index parameters. At the same time, optimization of the set of parameters that determine the waveguide becomes

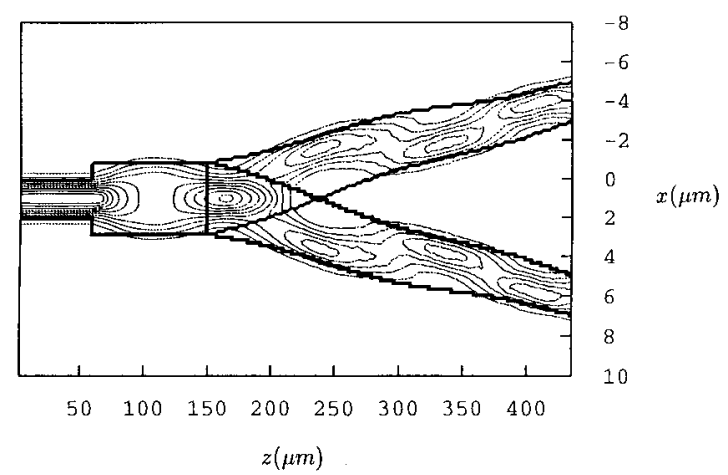

Fig. 8. Contour plots of the waveguide and the beam (optimized case) with two Fourier coefficients [MMI width, $3.75 \mu \mathrm{m}$; MMI length, $91.3 \mu \mathrm{m}$; $n$ (wedge), $\left.3.561 ; b_{1}=-0.15, b_{3}=0.16 ; \alpha=1\right]$. 


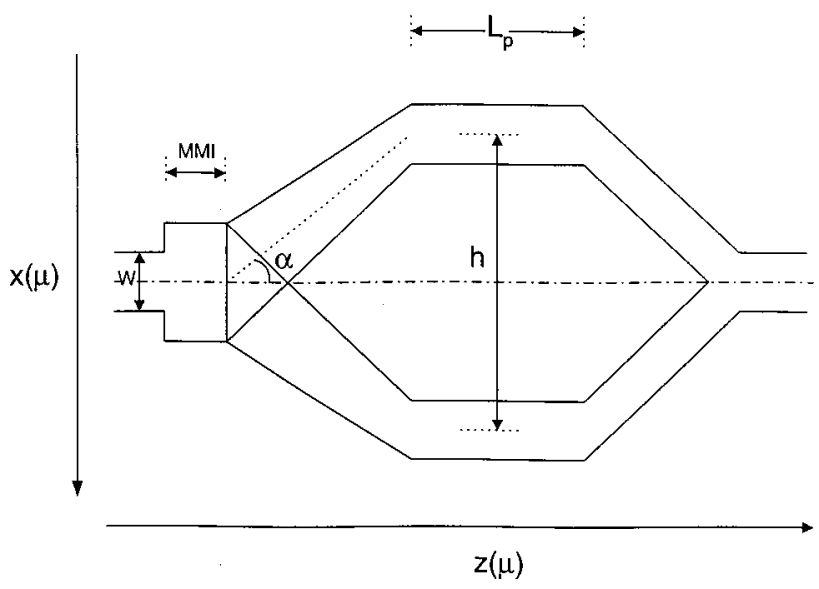

Fig. 9. Typical Mach-Zehnder waveguide.

more critical because small losses associated with each segment of a multistage device accumulate over many segments.

OCSD calculations for a five-parameter model of the Mach-Zehnder device shown in Fig. 9 were carried out. The initial conditions were determined as for the $Y$-junction case, but the length of the MMI splitter was taken as $55 \mu \mathrm{m}$, not $100 \mu \mathrm{m}$, to lessen the computational time. The initial values of the MMI width and the wedge index are $4 \mu \mathrm{m}$ and 3.558, respectively. The Fourier coefficients were initialized to zero corresponding to a straight waveguide. The length of the parallel arms $L_{p}$ was fixed at $200 \mu \mathrm{m}$. Similarly, the separated arm-to-arm distance $h$ in Fig. 9 was held fixed at a value of $10 \mu \mathrm{m}$.

For the Mach-Zehnder device, the target wave function is the same as the input mode wave function. The intensity plots of the output wave function and the target wave function for the unoptimized and the optimized cases for angle $\alpha=1$ are shown in Figs. 10 and 11. Here again, the output wave function and the target wave function are nearly indistinguishable

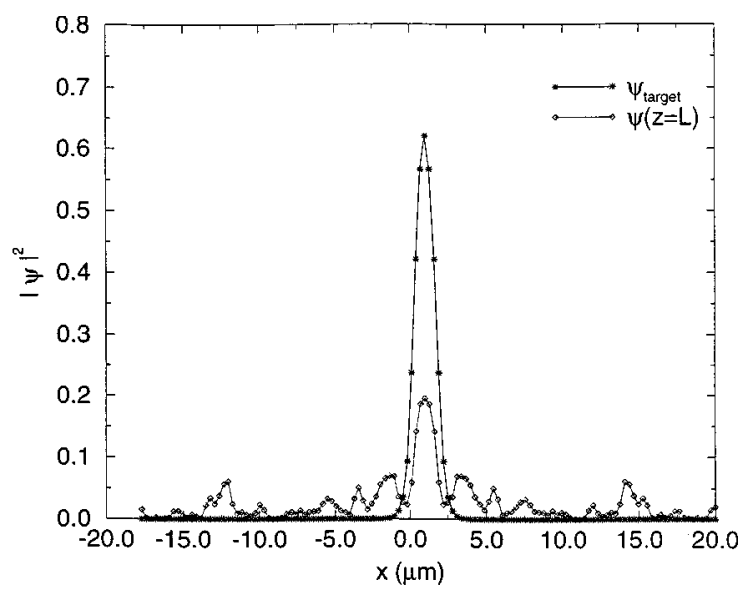

Fig. 10. Plot of the intensities of the wave function at $z=L$ (unoptimized case) and the target wave function for the MachZehnder device [MMI width, $4 \mu \mathrm{m}$; MMI length, $55 \mu \mathrm{m} ; n$ (wedge), 3.558; $\left.b_{2}=0.0, b_{3}=0.0 ; \alpha=1\right],\left|\left\langle\psi_{\text {target }} \mid \psi(z=L)\right\rangle\right|^{2}=0.26$.

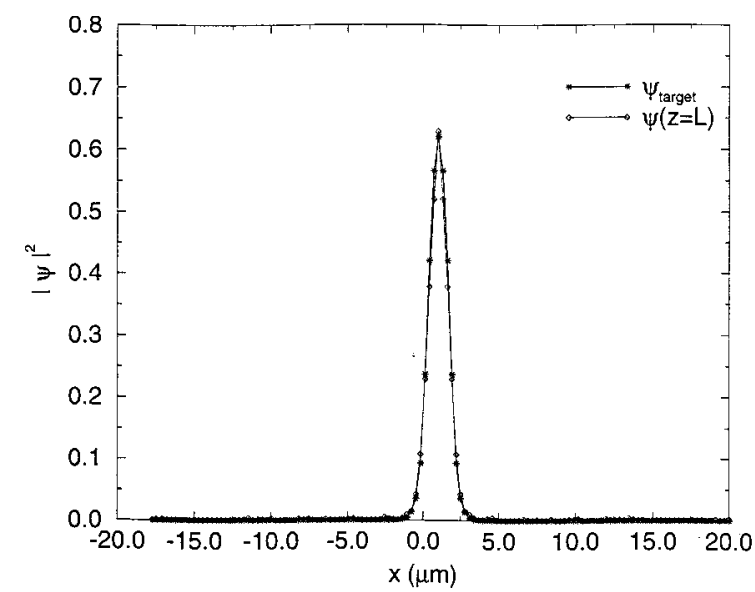

Fig. 11. Plot of the intensities of the wave function at $z=L$ (optimized case) and the target wave function for the Mach-Zehnder device [MMI width, $3.7 \mu \mathrm{m}$; MMI length, $66.2 \mu \mathrm{m}$; $n$ (wedge), 3.568; $\left.b_{2}=0.138, b_{3}=-0.137 ; \alpha=1\right],\left|\left\langle\psi_{\text {target }} \mid \psi(z=L)\right\rangle\right|^{2}=0.96$.

after the optimization. The corresponding contour plots are shown in Figs. 12 and 13. The values of the optimized parameters are a MMI width of $3.7 \mu \mathrm{m}$, MMI length of $66.2 \mu \mathrm{m}$, wedge index of $3.568, b_{2}=$ 0.138 , and $b_{3}=-0.137$. The longer MMI section and the shape of the $Y$ branches are clear in Fig. 13.

\section{Conclusions}

In this research we have shown that ideas of OCT developed in the field of quantum dynamics ${ }^{4}$ and recently adapted to optical waveguide $\operatorname{design}^{5}$ can be applied successfully to realistic branching waveguides. In particular, the OCT method, implemented within the framework of the paraxial equation, can treat arbitrary structural and refractive-index variations. Scaling of computational effort with number of parameters is mild; it should be possible to simultaneously optimize dozens of parameters on current work stations.

The improvement obtained by application of OCT relative to the performance of waveguides based on reasonable initial guess values for their variable

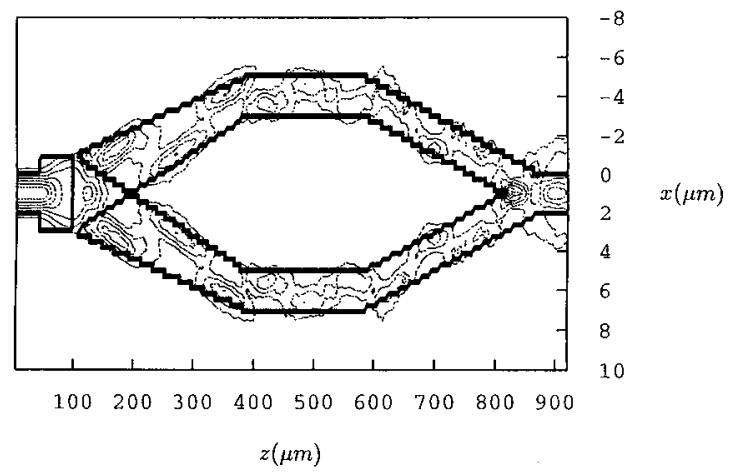

Fig. 12. Contour plots of the waveguide and the beam (unoptimized case) for the Mach-Zehnder device [MMI width, $4 \mu \mathrm{m}$; MMI length, $55 \mu \mathrm{m} ; n$ (wedge), 3.558; $\left.b_{2}=0.0, b_{3}=0.0 ; \alpha=1\right]$, $\left|\left\langle\psi_{\text {target }} \mid \psi(z=L)\right\rangle\right|^{2}=0.26$. 


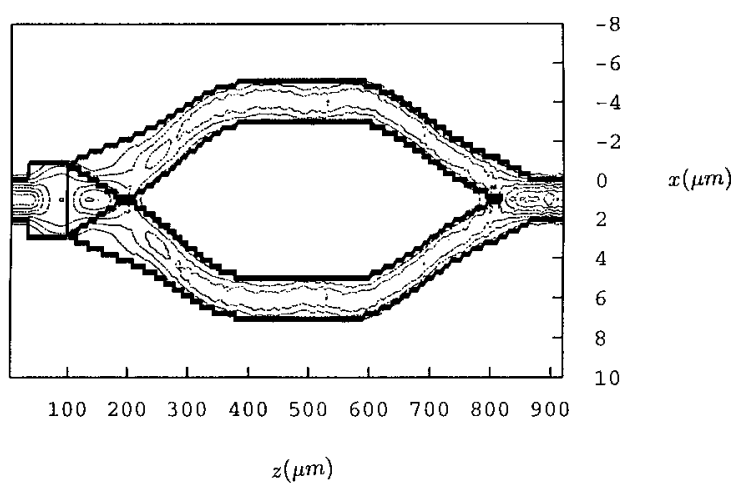

Fig. 13. Contour plots of the waveguide and the beam (optimized case) for the M-Z device [MMI width, $3.7 \mu \mathrm{m}$; MMI length, $66.2 \mu \mathrm{m} ; n$ (wedge), 3.568; $\left.b_{2}=0.138, b_{3}=-0.137 ; \alpha=1\right]$, $\left|\left\langle\psi_{\text {target }} \mid \psi(z=L)\right\rangle\right|^{2}=0.96$.

structural and index parameters can be significant. We found substantial improvement in guiding for both wide-angle $Y$ branches and corresponding Mach-Zehnder devices.

Outstanding theoretical problems include the extension of OCT to treat nonparaxial wave propagation. Interesting applications to complex structures, e.g., Mach-Zehnder devices with a perturbed index in one arm (to generate destructive interference in the recombining beams $)^{25,26}$ and staged, multicomponent waveguides, lie ahead.

We thank NATO for the financial support (grant CRG 941284) that made possible this collaboration. R. D. Coalson also acknowledges partial support from the National Science Foundation grant CHE-9529674.

\section{References and Notes}

1. J. D. Jackson, Classical Electrodynamics, 2nd ed. (Wiley, New York, 1975)

2. D. Marcuse, Theory of Dielectric Waveguides, 2nd ed. (Academic, New York, 1991)

3. See, for example, A. H. Cherin, An Introduction to Optical Fibers (McGraw-Hill, New York, 1983).

4. S. Shi and H. Rabitz, "Quantum mechanical optimal control of physical observables in microsystems," J. Chem. Phys. 92, 364-376 (1990); P. Gross, V. Ramakrishna, E. Vilallonga, H. Rabitz, M. Littman, S. A. Lyon, and M. Shayegan, "Optimally designed potentials for control of electron-wave scattering in semiconductor nanodevices," Phys. Rev. B 49, 11,100-11,110 (1994).

5. D. K. Pant, R. D. Coalson, M. I. Hernandez, and J. CamposMartinez, "Optimal control theory for the design of optical waveguides," J. Lightwave Technol. 16(2), 292-300 (1998).

6. D.-S. Min, D. W. Langer, D. K. Pant, and R. D. Coalson, "Wide angle low-loss waveguide branching for integrated optics," Fiber Integr. Opt. 16, 331-342 (1997).

7. D.-S. Min, "Channeling devices for high speed signals in integrated optics and circuits," Ph.D. dissertation (University of Pittsburgh, Pittsburgh, Pa., 1998).

8. O. Hanaizumi, M. Miyagi, and K. Kawakami, "Wide Y-junctions with low losses in three dimensional dielectric optical waveguides," IEEE J. Quantum Electron. QE-21(2), 168173 (1985).

9. M. H. Hu, J. Z. Huang, R. Scarmozzino, M. Levy, and R. M. Osgood, Jr., "A low-loss and compact waveguide Y-branch us- ing refractive index tapering," IEEE Photon. Technol. Lett. 9(2), 203-205 (1997).

10. H. Hatami-Hanza, P. L. Chu, and M. J. Lederer, "A new lowloss wide angle Y-branch configuration for optical dielectric slab waveguides," IEEE Photon. Lett. 6(4), 528-530 (1994).

11. H. P. Chan, S. Y. Cheng, and P. S. Chung, "Low-loss wideangle symmetric Y-branch waveguide," Electron. Lett. 32(7), 652-654 (1996); "Novel design of low-loss wide-angle asymmetric Y-branch waveguides," Microwave Opt. Technol. Lett. 111(2), 87-89 (1996).

12. L. B. Soldano and E. C. M. Pennings, "Optical multi-mode interference devices based on self-imaging: principles and applications," J. Lightwave Technol. 13(4), 615-627 (1995).

13. D. A. McQuarrie, Quantum Chemistry (University Science, Mill Valley, Calif., 1983); E. Merzbacher, Quantum Mechanics, 2nd ed. (Wiley, New York, 1970).

14. G. B. Hocker and W. K. Burns, "Mode dispersion in diffused channel waveguides by the effective index method," Appl. Opt. 16, 113-118 (1977).

15. K. S. Chiang, "Analysis of optical fibers by the effective index method," Appl. Opt. 25, 348-354 (1986).

16. Note that $V(x, z) \cong-k \Delta n(x, z)$, where $\Delta n(x, z) \equiv n_{\text {eff }}(x, z)-$ $n_{0}$ is the deviation of the index from the reference value $n_{0}$, which in the waveguides of interest here is much less than the reference value itself, i.e., $\Delta n / n_{0} \ll 1$.

17. M. D. Feit and J. A. Fleck, Jr., "Computation of mode properties in optical fiber waveguides by a propagating beam method," Appl. Opt. 19, 1154-1164 (1980).

18. J. Van Roey, J. van der Donk, and P. E. Lagasse, "Beam propagation method: analysis and assessment," J. Opt. Soc. Am. 71, 803-810 (1981).

19. Criteria other than output quality can in principle be included in the cost function. Some possibilities were considered in Ref. 5. Here for simplicity we assume that output quality is the only important issue.

20. Other criteria for optimal guiding are possible, for example, maximization of the integrated beam intensity within the boundaries of the guide at its output. There exists an appropriate projection operator analogous to the one given in Eq. (5) for each optimal guiding criterion.

21. W. H. Press, B. P. Flannery, S. A. Teukolsky, and W. T. Vetterling, Numerical Recipes in Fortran: The Art of Scientific Computing (Cambridge U. Press, Cambridge, UK, 1992).

22 . To check that the paraxial equation yields an accurate approximation to the full Helmholtz equation, we utilized the numerical method presented in Ref. 23 to solve the latter equation for several parameter sets considered in this paper. The results were found to be essentially identical to those obtained from the paraxial equation, giving us confidence that the paraxial equation suffices for the systems of interest here. Development of an OCT procedure for direct optimization of wave propagation according to the Helmholtz equation presents an interesting problem for further research.

23. S. Banerjee and A. Sharma, "Propagation characteristics of optical waveguiding structures by direct solution of the Helmholtz equation for total fields," J. Opt. Soc. Am. A 6, 1884-1894 (1989).

24. The calculation presented in Fig. 8, with five adjustable parameters, required approximately 50 iterations and took approximately $90 \mathrm{~min}$ of CPU time on a low-end work station.

25. J. E. Zucker, K. L. Jones, B. I. Miller, and U. Koren, "Miniature Mach-Zehnder InGaAsP quantum well waveguide interferometers for $1.3 \mu \mathrm{m}$," IEEE Photon. Technol. Lett. 2(1), 32-34 (1990).

26. J. S. Cites and P. R. Ashley, "High performance Mach-Zehnder modulators in multiple quantum well GaAs/AlGaAs," J. Lightwave Technol. 12, 1167-1173 (1994). 